\title{
Recognising the threat of vision loss in people living with HIV on antiretroviral therapy without retinitis
}

\begin{tabular}{|c|c|}
\hline \multicolumn{2}{|c|}{$\begin{array}{l}\text { Authors: } \\
\text { Alvin J. Munsamy }{ }^{1} \text { (D) } \\
\text { Anandan A. Moodley }^{2} \\
\text { Rune L. Brautaset }^{3}\end{array}$} \\
\hline \multicolumn{2}{|c|}{$\begin{array}{l}\text { Affiliations: } \\
{ }^{1} \text { Discipline of Optometry, } \\
\text { School of Health Science, } \\
\text { University of KwaZulu-Natal, } \\
\text { Durban, South Africa }\end{array}$} \\
\hline \multicolumn{2}{|c|}{$\begin{array}{l}\text { Department of Neurology, } \\
\text { Universitas Hospital, } \\
\text { University of the Free State, } \\
\text { Bloemfontein, South Africa }\end{array}$} \\
\hline \multicolumn{2}{|c|}{$\begin{array}{l}{ }^{3} \text { Division of Eye and Vision, } \\
\text { Department of Clinical } \\
\text { Neuroscience, Karolinska } \\
\text { Institutet, Stockholm, } \\
\text { Sweden }\end{array}$} \\
\hline \multicolumn{2}{|c|}{$\begin{array}{l}\text { Corresponding author: } \\
\text { Alvin Munsamy, } \\
\text { munsamya1@ukzn.ac.za }\end{array}$} \\
\hline \multicolumn{2}{|c|}{$\begin{array}{l}\text { Dates: } \\
\text { Received: } 13 \text { Nov. } 2019 \\
\text { Accepted: } 09 \text { Apr. } 2020 \\
\text { Published: } 22 \text { July } 2020\end{array}$} \\
\hline \multicolumn{2}{|c|}{$\begin{array}{l}\text { How to cite this article: } \\
\text { Munsamy AJ, Moodley AA, } \\
\text { Brautaset RL. Recognising } \\
\text { the threat of vision loss in } \\
\text { people living with HIV on } \\
\text { antiretroviral therapy without } \\
\text { retinitis. Afr Vision Eye } \\
\text { Health. 2020;79(1), a547. } \\
\text { https://doi.org/10.4102/ } \\
\text { aveh.v79i1.547 }\end{array}$} \\
\hline \multicolumn{2}{|c|}{$\begin{array}{l}\text { Copyright: } \\
\text { (C) 2020. The Authors. } \\
\text { Licensee: AOSIS. This wor } \\
\text { is licensed under the } \\
\text { Creative Commons } \\
\text { Attribution License. }\end{array}$} \\
\hline \multicolumn{2}{|l|}{ Read online: } \\
\hline 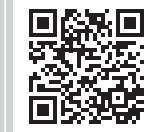 & $\begin{array}{l}\text { Scan this QR } \\
\text { code with your } \\
\text { smart phone or } \\
\text { mobile device } \\
\text { to read online. }\end{array}$ \\
\hline
\end{tabular}

Background: People living with HIV (PLHIV) on antiretroviral therapy (ART) without retinitis may not have a healthy retinal structure.

Aim: To examine the impact of the virus together with the ART medication in PLHIV without retinitis.

Methods: This review used the following databases: PubMed, Google Scholar and EBSCOhost. Search terms included: HIV and/or retinal nerve fibre layer (RNFL); perimetry; colour vision; contrast sensitivity (CS); visual evoked potentials (VEPs); electroretinograms (ERGs); and the brain. All peer-reviewed studies related to PLHIV on ART without retinitis, up until September 2019, were included.

Results: The mean RNFL thickness, and superior and inferior zones showed thinning. Affected visual functions include transient pattern VEP and ERG; contrast sensitivity; reduced total error scores on colour vision evaluation; and reduced mean deviation and pattern standard deviations on perimetry. Studies also showed concurrent thinning of the peripapillary retinal nerve fibre layer (ppRNFL) and perimetry, contrast sensitivity and colour vision. All these significant observations were seen at a cluster of differentiation 4 (CD4) count of less than 200 cells $/ \mathrm{mm}^{3}$ in PLHIV on ART with no retinitis. Further to this, studies have also related the retina to grey and white matter changes in PLHIV in the era of ART. A gap in research involves studies on the vascular impact of ART on the retina, which should be factored into studies going forward when studying PLHIV on ART.

Conclusion: The decrease in visual function, the RNFL changes and neuro-ophthalmic involvement in PLHIV allow us to recognise the threat to vision loss when factoring in the expected longer lifespan that PLHIV on ART are afforded today.

Keywords: HIV; retinal nerve fibre layer; vision; contrast sensitivity; visual electrophysiology; colour vision; neuro-ophthalmic; ART.

\section{Background}

Should we examine the impact of this virus, with its treatment of the eyes of people living with HIV (PLHIV), until such time as a vaccine is found? The 'test and treat' approach makes it possible to preserve the lives of PLHIV with universal access to antiretroviral therapy (ART). It is the driving force for reduced episodes of retinitis in PLHIV by elevating cluster of differentiation 4 (CD4) counts and reducing viral loads. All of this is possible as a result of the Joint United Nations Programme on HIV / AIDS (UNAIDS) goal of 90-90-90, which suggests that this disease and its treatment cannot be separated from targeting $90 \%$ of PLHIV who are diagnosed, and who are on ART, and achieve viral suppression by 2020. South Africa has the largest ART programme in the world with $68 \%$ of its $7.7 \mathrm{~m}$ HIV sufferers on ART, which raised their life expectancy to 67.7 years in $2015 .^{1}$ This translates to $62 \%$ of all PLHIV on ART worldwide.

Ophthalmic clinicians need to be aware that while PLHIV on ART may not show clinical signs of eye deterioration, which usually is indicated by a lack of evidence of retinitis, as a result of the benefits of ART, this does not mean that the retinal structure is healthy. Presently, ophthalmic clinicians manage HIV only when the eye shows clinical signs of deterioration. When vision is threatened, the retina may be affected by showing retinitis, in particular cytomegalovirus (CMV) retinitis. All of this usually occurs when PLHIV have CD4 counts of less than 50 cells $/ \mathrm{mm}^{3}$. As the current standard practice is for PLHIV to be initiated onto ART upon diagnosis, there is a reduced prevalence of opportunistic infections, including those that affect the eyes. However, this review of relevant research argues that there is evidence that ophthalmic clinicians need to adopt a proactive approach in managing the eyes of PLHIV who are on ART, until such time as a vaccine 
is found. Understanding this will help clinicians to better manage the threat of blindness in PLHIV.

This review attempts to highlight the structural integrity of the retinal nerve fibre layer (RNFL) in PLHIV who are on ART without retinitis. The RNFL is a fundamental layer of the inner retina, as it conducts most of our visual functions including visual fields (perimetry), contrast sensitivity (CS), colour vision and visual electrophysiology. The integrity of these visual functions will be explored, including the existence of a structural relationship with the RNFL to assess its impact in the absence of retinitis.

The review will first discuss studies on RNFL thickness in PLHIV, then describe studies focusing on visual function assessment in PLHIV, and then describe studies that have assessed the RNFL in the presence of various visual functional changes in PLHIV, as an attempt to demonstrate a direct relationship between the RNFL and visual changes. Finally, it will explore neuro-ophthalmic associations in PLHIV and conclude with the effects of ART itself on the retina. The value of this review is to alert practitioners to the structural and functional integrity of the retina and its impact on vision in this focus group of PLHIV on ART without retinitis.

\section{Methods}

The literature search was performed for this review using the PubMed, Google Scholar and EBSCOhost platforms' databases published up until September 2019. The search terms used were HIV and RNFL, HIV and perimetry, HIV and contrast sensitivity, HIV and colour vision, HIV and visual evoked potentials, HIV and electroretinograms, HIV and brain, $H I V$ and ART, and retina. The inclusion criteria for the articles were all types of peer-reviewed article related to PLHIV on ART without retinitis. The exclusion criteria were all grey literature. The methodology includes title screening followed by abstract screening and full text screening for each database. Literature was then categorised according to their different foci on issues related to the purpose of the study. The categorisations and publications that were found and reviewed are presented in Tables 1, 2, 3 and 4 .

\section{Ethical considerations}

The study has received ethical clearance from the Biomedical Research Ethics Committee, University of KwaZulu-Natal (ethical clearance number BE359/17). Informed consent was obtained from all participants prior to any form or part of the data collection.

\section{Results \\ Retinal nerve fibre layer in people living with HIV}

The RNFL is the innermost layer of the retina comprising axons of the retinal ganglion cells, with a mean RNFL thickness in adults of approximately 100 microns. However, variations in RNFL thickness are associated with population genotype differences; in short, with race, sex and age. Thinning of the RNFL, ganglion cell layer (GCL), and of the retina generally, are a physiological change of ageing.

TABLE 1: Studies investigating retinal nerve fibre layer thickness in people living with HIV on antiretroviral therapy without retinitis.

\begin{tabular}{|c|c|c|c|c|c|c|}
\hline RNFL/Retina & Study & Tool & Sample size $(n)$ & CD4 count & RNFL/Retinal thickness $(\mu \mathrm{m})$ & Statistical significance \\
\hline \multirow[t]{2}{*}{ ppRNFL } & \multirow[t]{2}{*}{ Plummer et al. $^{2}$} & \multirow[t]{2}{*}{ HRT } & 17 & $H I V(+)$ & Mean RNFL $=230$ & \multirow[t]{2}{*}{$p=0.011$} \\
\hline & & & 24 & HIV(-) & Mean RNFL = 280 & \\
\hline \multirow[t]{3}{*}{ ppRNFL } & \multirow[t]{3}{*}{ Kozak et al. ${ }^{3}$} & \multirow{3}{*}{$\begin{array}{l}\text { Stratus OCT } \\
(3000)\end{array}$} & 18 & Nadir CD4 > $100(\mathrm{HIV}+)$ & Mean overall RNFL $=103.30 \pm 9.28$ & \multirow[t]{3}{*}{$p<0.001$} \\
\hline & & & 25 & Nadir CD4 < $100(\mathrm{HIV}+)$ & Mean overall RNFL = $90.1 \pm 12.5$ & \\
\hline & & & 22 & $\operatorname{HIV}(-)$ & Mean overall RNFL $=103.33 \pm 8.5$ & \\
\hline \multirow[t]{4}{*}{ ppRNFL } & \multirow[t]{4}{*}{ Besada et al. ${ }^{5}$} & \multirow[t]{2}{*}{ HRT } & 13 & Nadir CD4 > 100 & Mean RNFL $=230 \pm 40$ & \multirow[t]{2}{*}{$p<0.05$} \\
\hline & & & 13 & HIV(-) & Mean RNFL $=290 \pm 70$ & \\
\hline & & \multirow[t]{2}{*}{ GDx } & 12 & $H I V(+)$ & $\begin{array}{l}\text { Mean superior } \max =77.17 \pm 10.45 \\
\text { Mean inferior } \max =77.60 \pm 11.52\end{array}$ & \multirow[t]{2}{*}{$p<0.05$} \\
\hline & & & 10 & HIV(-) & $\begin{array}{l}\text { Mean superior } \max =87.34 \pm 7.93 \\
\text { Mean inferior } \max =87.18 \pm 8.78\end{array}$ & \\
\hline \multirow[t]{6}{*}{ ppRNFL } & \multirow[t]{6}{*}{ Kozak et al. ${ }^{4}$} & \multirow[t]{6}{*}{ GDx-VCC } & \multirow[t]{2}{*}{26} & \multirow[t]{2}{*}{ Nadir CD4 > 100} & Superior average $=66 \pm 7$ & $\begin{array}{l}p>0.05 \\
\operatorname{HIV}(+) \text { VS HIV(-) }\end{array}$ \\
\hline & & & & & Inferior average $=64 \pm 8$ & $\begin{array}{l}p<0.05 \\
\operatorname{HIV}(+)>100 \text { vs } \operatorname{HIV}(+)<100\end{array}$ \\
\hline & & & \multirow[t]{2}{*}{35} & \multirow[t]{2}{*}{ Nadir CD4 $<100$} & Superior average $=63 \pm 11$ & $\begin{array}{l}p<0.05 \\
\text { HIV }+) \text { vs HIV(-) and } \\
>100 \text { vs }<100\end{array}$ \\
\hline & & & & & Inferior average $=60 \pm 10$ & $\begin{array}{l}p<0.05 \\
\text { HIV(+) vs HIV(-) and } \\
>100 \text { vs }<100\end{array}$ \\
\hline & & & \multirow[t]{2}{*}{25} & \multirow[t]{2}{*}{ HIV $(-)$} & Superior average $=68 \pm 7.5$ & $p<0.05 \mathrm{HIV}(+)$ vs HIV(-) and $>100$ vs $<100$ \\
\hline & & & & & Inferior average $=64 \pm 6.1$ & $p<0.05 \mathrm{HIV}(+)$ vs HIV(-) and $>100$ vs $<100$ \\
\hline \multirow[t]{2}{*}{ Total retina (fovea) } & \multirow[t]{2}{*}{ Arcinue et al. ${ }^{6}$} & \multirow{2}{*}{$\begin{array}{l}\text { Spectralis } \\
\text { OCT }\end{array}$} & 10 & $\mathrm{CD} 4<100$ & Mean thickness $=232.6 \pm 23.4$ & \multirow[t]{2}{*}{$p<0.05$} \\
\hline & & & 10 & HIV(-) & Mean thickness $=213.1 \pm 14.5$ & \\
\hline \multirow[t]{2}{*}{ Total retina(macula) } & & & - & $\mathrm{CD} 4<100$ & Mean thickness $=268.5 \pm 20.3$ & \multirow[t]{2}{*}{$p<0.05$} \\
\hline & & & - & HIV(-) & Mean thickness $=244.1 \pm 11.2$ & \\
\hline \multirow[t]{2}{*}{ GCL (macula) } & & & - & $\mathrm{CD} 4<100$ & Mean thickness $=19.0 \pm 12.7$ & \multirow[t]{2}{*}{$p<0.05$} \\
\hline & & & - & HIV $(-)$ & Mean thickness $=9.3 \pm 4.5$ & \\
\hline
\end{tabular}

ppRNFL, peripapillary retinal nerve fibre layer; GCL, ganglion cell layer; RNFL, retinal nerve fibre layer; CD4, cluster of differentiation 4; GDx nerve fibre layer analyser; GDx-VCC, GDx variable corneal compensation. 
TABLE 2: Studies investigating visual function in people living with HIV on antiretroviral therapy with no retinitis.

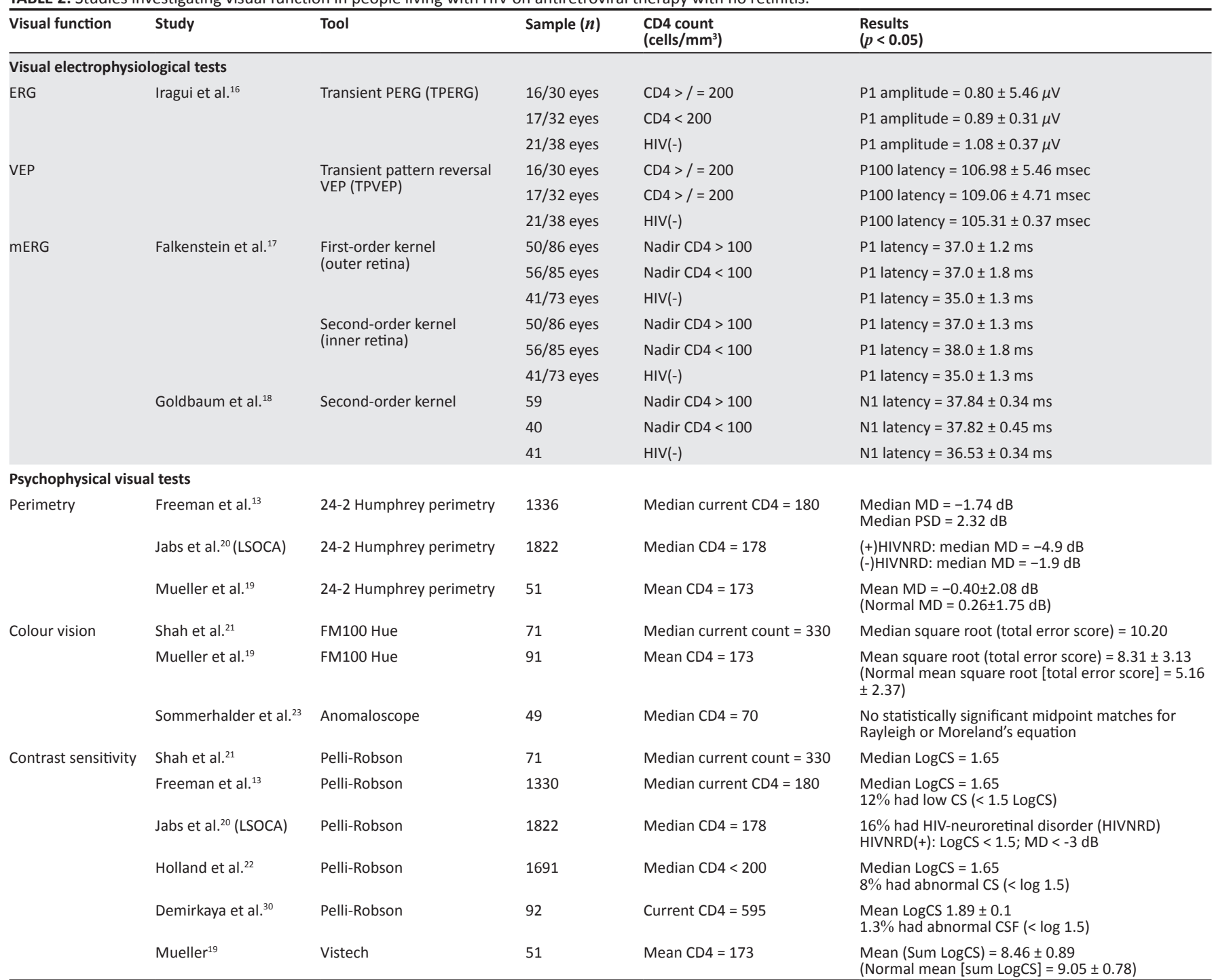

HIVNRD, HIV as a neuroretinal disorder; LogCS, log unit contrast sensivity; ERG, electroretinogram; VEP, visual evoked potential; mERG, multifocal electroretinogram; CD4, cluster of differentiation 4; PERG, pattern electroretinogram; MD, mean deviation; PSD, pattern standard deviation; dB, decibel.

There is evidence that supports the associations of HIV infection and ART with changes in the retina and the effects on the RNFL. Despite studies comprising small patient sample sizes, and with varied results amongst different populations of PLHIV, Table 1 shows a thinning of the peripapillary retinal nerve fibre layer (ppRNFL) in PLHIV without retinitis and, furthermore, makes a case for questioning if the RNFL thickness can also be a structural biomarker for visual function in HIV.

Plummer et al., ${ }^{2}$ in a comparative study, investigated HIV-positive patients without CMV retinitis on ART and HIV-negative persons. Using the Heidelberg Retinal Tomograph (HRT), they noted a loss of retinal ganglion cells and concluded that there is a thinning of the RNFL without an infectious retinopathy. Kozak et al., ${ }^{3}$ also using a comparative study with HIV-negative persons, likewise showed RNFL loss in PLHIV without CMV retinitis while on ART for 6 months, but at CD4 counts of not below 100 cells $/ \mathrm{mm}^{3}$. Kozak et al., ${ }^{4}$ in 2007 , also reported a reduction in ppRNFL thickness in subjects with CD4 counts of less than
100 cell $/ \mathrm{mm}^{3}$. Besada et al. ${ }^{5}$ also observed a reduction of ppRNFL thickness but in subjects with CD4 counts of greater than 100 cells $/ \mathrm{mm}^{3}$. All of this growing evidence suggests that in the absence of clinically significant retinal signs, the RNFL is affected in PLHIV on ART but with CD4 counts considered to indicate an immunocompromised state.

Table 1 shows the RNFL thickness measurements using different tools from the above-mentioned studies. It specifically shows the effects on mean ppRNFL thickness and furthermore highlights the thinning in superior and inferior ppRNFL thickness. The different tools used makes it challenging to directly compare the measurements, but two studies, performed by Plummer et al. ${ }^{2}$ and Besada et al. ${ }^{5}$ using HRT, showed identical thinning with a mean ppRNFL of 230 microns in PLHIV with minimum CD4 counts of 100 cell/ $\mathrm{mm}^{3}$ while on ART when compared to controls. However, this empirical evidence of the integrity of the RNFL is limited even while on ART because it occurs at low immunocompetence. Accordingly, these studies do not focus on higher immunocompetence (higher CD4 counts), which may be more 
TABLE 3: Studies investigating the relationship between the retinal nerve fibre layer and visual function in people living with HIV on antiretroviral therapy with no retinitis.

\begin{tabular}{|c|c|c|c|c|c|c|}
\hline Vision function/RNFL & Study & Tool & Sample $(n)$ & CD4 count (cell/mm ${ }^{3}$ ) & Associations & Statistical significance \\
\hline \multirow[t]{8}{*}{$\begin{array}{l}\text { Contrast sensitivity } \\
\text { and ppRNFL }\end{array}$} & Kalyani et al. ${ }^{12}$ & $\begin{array}{l}\text { Pelli-Robson; } \\
\text { RTVue OCT }\end{array}$ & 57 & $\begin{array}{l}\text { Median current } C D 4=420 \\
\text { Median nadir } C D 4=66\end{array}$ & $\begin{array}{l}\text { Temporal RNFL thickness positively } \\
\text { correlated with worse LogCS }\end{array}$ & $r=0.295, p=0.003$ \\
\hline & \multirow[t]{4}{*}{ Pathai et al. ${ }^{27}$} & \multirow{4}{*}{$\begin{array}{l}\text { Pelli-Robson; } \\
\text { Spectral-domain OCT }\end{array}$} & 216 & Median current CD4 $=468$ & \multirow{4}{*}{$\begin{array}{l}\text { LogCS } 1.70 \text { was associated with } \\
\text { thin temporal RNFL }\end{array}$} & \multirow[t]{4}{*}{ O.R. $=3.39, p=0.02$} \\
\hline & & & 215 & HIV(-) & & \\
\hline & & & 225 & Median nadir CD4 = 136 & & \\
\hline & & & 203 & HIV(-) & & \\
\hline & Paul et al. ${ }^{28}$ & $\begin{array}{l}\text { Pelli-Robson } \\
\text { (smartphone); } \\
\text { Spectralis OCT }\end{array}$ & 17 & Mean CD4 $=527 \pm 290$ & $\begin{array}{l}\text { Temporal RNFL thickness } \\
\text { positively correlated with LogCS }\end{array}$ & $r=0.37, p=0.002$ \\
\hline & \multirow[t]{2}{*}{ Demirkaya et al. ${ }^{30}$} & \multirow[t]{2}{*}{$\begin{array}{l}\text { Pelli-Robson; } \\
\text { Topcon 3D OCT }\end{array}$} & 92 & $\begin{array}{l}\text { Nadir CD4 count }=180 \\
\text { Current } C D 4 \text { count }=595\end{array}$ & \multirow{2}{*}{$\begin{array}{l}\text { No clinically relevant reduction of } \\
\text { CS and the absence of } \\
\text { neuroretinal atrophy }\end{array}$} & \multirow[t]{2}{*}{$\mathrm{n} / \mathrm{a}$} \\
\hline & & & 63 & HIV(-) & & \\
\hline \multirow[t]{3}{*}{ Visual acuity and RNFL } & \multirow[t]{3}{*}{ Bartselli et al. ${ }^{29}$} & \multirow{3}{*}{$\begin{array}{l}\text { ETDRS chart/ } \\
\text { simulator; } \\
\text { Spectralis OCT }\end{array}$} & 19 & Nadir CD4 > 200 & \multirow{3}{*}{$\begin{array}{l}\text { Reduced visual acuity (at varying } \\
\text { contrast and luminance) was } \\
\text { associated with thinning of RNFL } \\
\text { in the temporal-inferior sector }\end{array}$} & \multirow[t]{3}{*}{$p<0.05$} \\
\hline & & & 28 & Nadir CD4 $<200$ & & \\
\hline & & & 57 & HIV $(-)$ & & \\
\hline \multirow{4}{*}{ Perimetry and ppRNFL } & \multirow{2}{*}{ Arantes et al. ${ }^{25}$} & \multirow{2}{*}{$\begin{array}{l}\text { Humphrey matrix } \\
\text { frequency doubling } \\
\text { technology (FDT); } \\
\text { Stratus OCT }\end{array}$} & 25 & CD4 $>100$ & \multirow{2}{*}{$\begin{array}{l}\text { Eyes with GHT and PSD outside } \\
\text { normal limits had significantly } \\
\text { thinner average ppRNFL }\end{array}$} & \multirow{2}{*}{$\begin{array}{l}\mathrm{GHT}: p=0.032 \\
\mathrm{PSD}: p=0.014\end{array}$} \\
\hline & & & 22 & HIV(-) & & \\
\hline & \multirow[t]{2}{*}{ Arantes et al. ${ }^{26}$} & \multirow{2}{*}{$\begin{array}{l}\text { Humphrey matrix } \\
\text { frequency doubling } \\
\text { technology (FDT); } \\
\text { Stratus OCT }\end{array}$} & 51 & Median current $\mathrm{CD} 4=355.48$ & $\begin{array}{l}\text { In PLHIV PSD and the average } \\
\text { ppRNFL had an association }\end{array}$ & $r^{2}=0.0185, p=0.002$ \\
\hline & & & 22 & HIV(-) & $\begin{array}{l}\text { Strongest correlations: } \\
\text { - between superior RNFL and } \\
\text { inferior visual field zones } \\
\text { - between nasal RNFL measures } \\
\text { and the temporal visual field zone }\end{array}$ & $\begin{array}{l}r^{2}=0.252, p<0.001 \\
r^{2}=0.171, p=0.003\end{array}$ \\
\hline $\begin{array}{l}\text { Colour vision and } \\
\text { ppRNFL }\end{array}$ & Kalyani et al. ${ }^{11}$ & $\begin{array}{l}\text { Lanthony D15; } \\
\text { RTVue OCT }\end{array}$ & 57 & $\begin{array}{l}\text { Median current } C D 4=420 \\
\text { Median nadir CD4 }=66\end{array}$ & $\begin{array}{l}\text { Temporal RNFL thickness } \\
\text { inversely correlated to CCI }\end{array}$ & $r=-0.338, p=0.0005$ \\
\hline
\end{tabular}

RNFL, retinal nerve fibre layer; ppRNFL, peripapillary retinal nerve fibre layer; CD4, cluster of differentiation 4; LogCS, log unit of contrast sensitivity; GHT, Glaucoma Hemifield Test; PSD, pattern standard deviation; r, correlation co-efficient; O.R., odds ratio.

applicable for the present as PLHIV are living longer and in a healthier state as a result of adhering to ART, which elevates the CD4 count and suppresses the viral load.

More recent research has indicated a different threat to the structural integrity of the eye in PLHIV by drawing attention for the first time to a thickening at the macula in contrast to ppRNFL thinning in PLHIV. Arcinue et al., ${ }^{6}$ using a comparative study of HIV-positive and HIV-negative persons, noted a reduction in cone density of $20 \%-30 \%$ at the macula in six subjects with CD4 counts of less than 100 cells $/ \mathrm{mm}^{3}$ while on ART. Moreover, an increase in thickness of 24.4 microns in the total macula, of 8.6 microns in the inner retina and 9.7 microns in the ganglion cell layer, was also found. The suggested cause was that of inner retinal oedema secondary to the retinovascular disease in HIV. The importance of the macula to central vision is fundamental and the suggestion of macula oedema does infer an impact on central vision; however, the level of effect is not discussed.

There is debate about the underlying causes of these structural changes to the eye amongst PLHIV. It has been suggested that HIV disrupts microvascular circulation in the eye which otherwise maintains RNFL integrity. ${ }^{5}$ There is a propensity with HIV infection for damage towards the central anatomical structures of the retina, specifically the posterior pole (optic nerve and macula) and major vascular arcades. ${ }^{4}$ This is supported by the studies shown in Table 1 that evaluate changes in ppRNFL changes and macula thickness. A competing explanation for RNFL changes in PLHIV is the stimulation of cytokine release syndrome which leads to the death of retinal ganglion cells. ${ }^{2}$ Ongoing replication of HIV itself causes apoptotic changes in specific neural tissues by stimulation of cytokine release (CR) by the immune system.

There is evidence that provides tangential support for the CR hypothesis; autopsies of HIV patients revealed axonal loss in the optic nerve. ${ }^{7}$ Secondary axonal loss results from damage to retinal ganglion cells due to the release of neurotoxins. Loss of myelinated optic nerve fibres is perhaps secondary to inner retinal damage, directly or indirectly resulting from HIV. The axonal degeneration may be a part of a wider neuroretinal degeneration in HIV, which postulates this form of pathogenesis of HIV as a neuroretinal disorder and not an isolated retinal disorder.

Proposed pathogenesis suggests that PLHIV may have accelerated ageing in comparison to HIV-negative persons. ${ }^{8,9}$ The hypothesis is that the final common pathway of accelerated ageing (immunosenescence) occurs as a result of mitochondrial toxicity, para-inflammation (which is a lowgrade inflammatory response to tissue stress or dysfunction associated with ageing), microvasculopathy and rheological abnormalities (caused by HIV, ART, genetic factors and associated risk factors such as injection drug use, co-infection and race). ${ }^{10}$

The use of ART in these studies also requires consideration. Colas et al. ${ }^{11}$ showed an increase in the RNFL thickness in PLHIV after 1 year of ART, while Kalayani et al. ${ }^{12}$ reported that the thinning of inferior and nasal RNFL was associated with the length of time an individual had been on ART notably a minimum of 180 months, therefore suggesting that a longer duration on ART may cause changes. All studies reviewed about PLHIV and on ART showed RNFL changes 


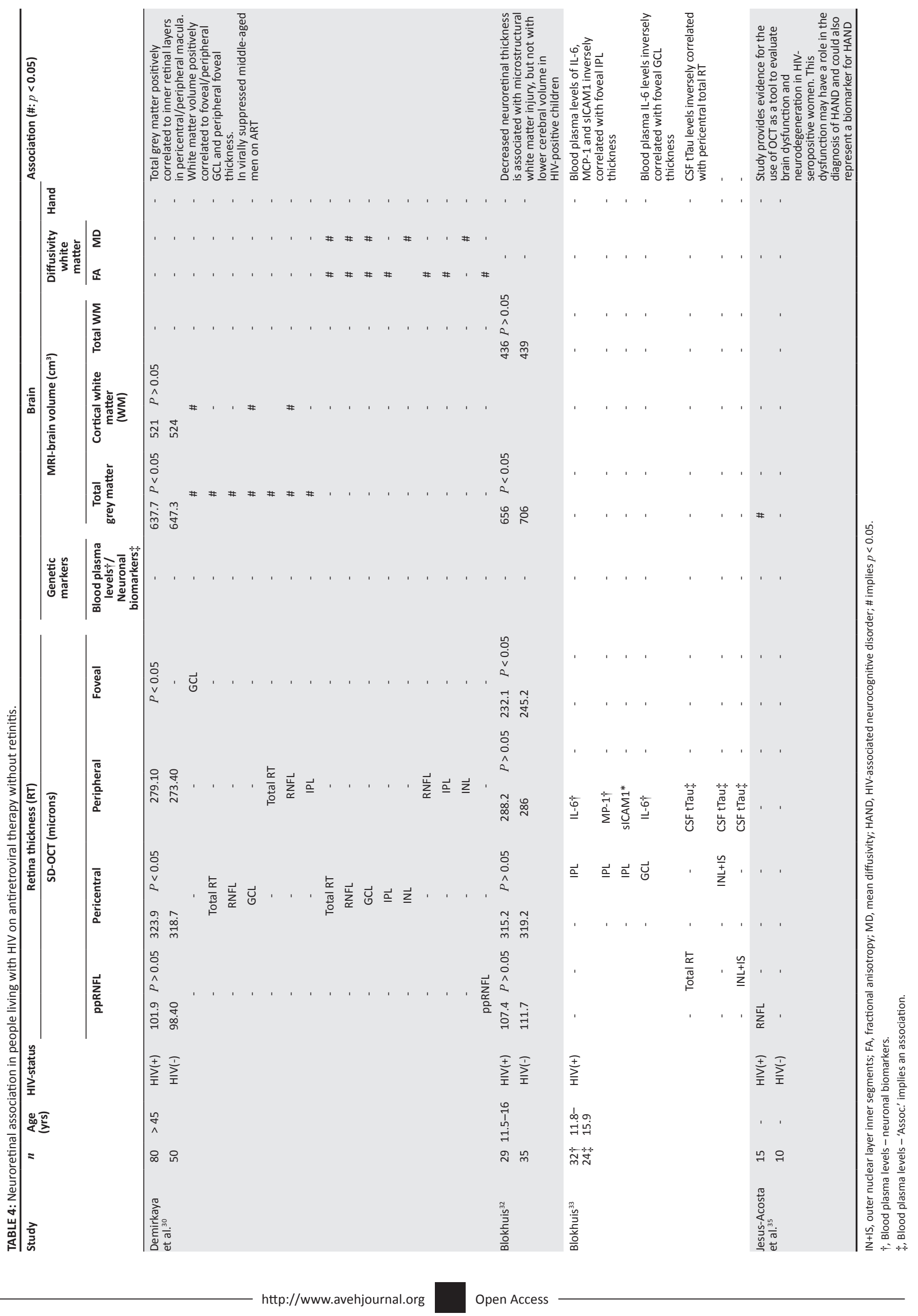


but these two studies highlighted the impact of ART on the RNFL, factoring its duration which, according to these studies, may be inversely proportional to treatment. Going forward, in studies evaluating PLHIV and the retina, it is necessary to take ART into account.

At present it can be stated with some confidence that changes to the RNFL is evidence that HIV infection itself forms part of the pathogenesis of neuroretinal dysfunction in PLHIV. $3,12,13$ The RNFL thins in PLHIV without retinitis and as we cannot stop ART, we should not ignore the threat of RNFL loss over time in PLHIV in light of the extended lifespan afforded by ART.

\section{Visual function in people living with HIV}

This review will now describe results from studies that have investigated changes in vision functionality in PLHIV. This is defined as visual electrophysiology and aspects of visual function that communicate physical changes occurring in the vision of PLHIV, referred to as psychophysical visual changes. The RNFL-focused research described thus far is the fundamental research to date that provides evidence of a structural change in the eye for understanding vision changes amongst PLHIV, particularly vision loss that is present when there is no active retinitis present. The following evidence presented has shown on electrophysiology that there are changes in visual function in PLHIV on ART without retinitis. Table 2 shows the aspects of visual functions affected and include visual evoked potential (VEP) and multifocal electroretinography (mERG) as well as psychophysical visual changes, namely CS, perimetry and colour vision.

\section{Visual electrophysiology in people living with HIV}

Electrophysiological techniques such as VEP and electroretinograms convey the integrity of the transmissions of the signals from the eye to the brain at different levels of transmission. Any changes in these electrical transmissions independently and objectively affirm clinical signs of damage in the retina and visual pathway, which may aid in a timeous diagnosis.

The pattern visual evoked potential (PVEP) is designed to establish the functional integrity of the entire visual pathway, while the pattern electroretinogram (PERG) assesses the functional integrity of the inner retina. These are observed at a sensitivity beyond funduscopic examination, which refers to the examination of the physical integrity of the inner eye, namely the retina and optic nerve. Axonal pathology in the optic nerve can be recognised by VEP independent of clinical signs and detectable morphology. ${ }^{14}$ The full field monocular PVEP P100 peak shows activation of the primary visual cortex (V1) and illustrates integrity of the pre-chiasmatic pathway. ${ }^{14}$

Both VEP and PERG have shown defects in activity of the ganglion cell layer in PLHIV without retinitis. ${ }^{15}$ This suggests that beyond psychophysical visual tests, the integrity of the visual function in PLHIV on ART without retinitis is affected.

Iragui et al. ${ }^{16}$ found that in PLHIV with a CD4 count of less than 200 cells $/ \mathrm{mm}^{3}$ there was reduced P1 transient PERG amplitude, implying ganglion cell dysfunction, as well as latency delay of the transient PVEP (P100), implying that this extends to the visual pathway. Furthermore, PLHIV with a CD4 count of greater than or equal to 200 cells $/ \mathrm{mm}^{3}$ showed a reduced amplitude of the transient PERG P1 potential. This establishes the dysfunctional changes at low CD4 counts in the visual pathway in PLHIV on ART without retinitis, as shown in Table 2.

Multifocal ERG is valuable in assessing visual function when the retina appears normal, as in this case of no retinitis in PLHIV. It can further distinguish retinal from optic nerve changes and most specifically focal retinal damage. In mERG studies, the first-order kernel represents outer retinal function whilst the second-order kernel is representative of inner retinal function.

Studies using mERG found reduced amplitudes and delayed latencies functionally; whilst this may not translate to the direct impact on the quality of life, it does show the impaired function of the inner retina and the visual pathway at low CD4 counts in PLHIV.

Falkenstein et al., ${ }^{15}$ using only mERG, suggests that the outer retina, which contains the photoreceptors, is quiescient in PLHIV on ART without retinopathy. Falkenstein et al. ${ }^{17}$ went on to show that HIV's effects are subtle in amplitudes between PLHIV with nadir CD4 counts of less than and greater than 100 cell $/ \mathrm{mm}^{3}$ for both kernels; but found widespread delays in implicit times or latent periods in both kernels for nadir CD4 counts of less than or greater than 100 cells $/ \mathrm{mm}^{3}$. Goldbaum et al. ${ }^{18}$ went further to show that apart from second-order kernels in mERG being a sensitive detector for inner retinal abnormalities, it separated HIV-positive eyes from normal eyes by showing the delayed implicit times in b-latency of the second-order kernels of mERG, thus showing impaired transmission of electrical stimuli. All this highlights that PLHIV on ART without retinopathy had retinal-processing abnormalities in both prolonged immunosuppression and PLHIV who have never been compromised immunologically.

Table 2 summarises, in PLHIV on ART without retinitis, that: the amplitude of transient PERG is less than $0.89 \mu \mathrm{V}$; the latency P100 in transient PVEP is greater than 106.98 ms; the P1 latency of the first-order kernel is delayed compared to HIV-negative persons; and that for the second-order kernel the P1 and b-latencies are prolonged. These electrophysiological changes provide evidence that, functionally, the retina is compromised in PLHIV on ART without retinitis, at low CD4 counts. What is yet to be proven is if this dysfunction extends to patients with higher CD4 counts, in light of the fact that PLHIV today live longer and 
in an immunocompetent state. The threat lies with the identified dysfunction compounding over time, translating into quality of life changes.

\section{Psychophysical visual changes (perimetry, colour vision and contrast sensitivity) in people living with HIV}

Exploratory studies in the last two decades have sought to find the underlying causes of visual changes amongst PLHIV. These studies first reported associations between HIV infection and the erosion of visual functionalities amongst PLHIV.

Psychophysical assessments refer to the analysis of visual function changes such as visual fields (perimetry), the effect on visual acuity (CS vision) and colour vision.

Perimetry (visual field) in people living with HIV: As early as 1997, Mueller et al. ${ }^{19}$ found abnormal changes using achromatic automated perimetry in PLHIV. These changes were at a mean CD4 count of 173 cells $/ \mathrm{mm}^{3}$ in PLHIV with $20 / 20$ visual acuity and no retinitis. Specifically, it was showed that $21.6 \%$ of PLHIV had a mean deviation of less than $-2.10 \mathrm{~dB}$ and that $27.5 \%$ had a GHT 'outside normal limits'. These findings suggest global and local changes in visual fields, where global or general loss of the visual field is represented by mean deviation scores, and sectoral loss of the visual fields by GHT. In 2008, Freeman et al. ${ }^{13}$ went on to find that $39 \%$ of PLHIV had abnormal mean deviations of less than $-2.63 \mathrm{~dB}$ and $33 \%$ had abnormal pattern standard deviation (PSD) of greater than $2.57 \mathrm{~dB}$, in a sample with a median current CD4 count of 180 cells $/ \mathrm{mm}^{3}$. Despite the mean versus median CD4 counts in these studies, both are in agreement that in immunocompromised PLHIV (with a CD4 count of less than 200 cells $/ \mathrm{mm}^{3}$ ) there are losses, albeit subtle, demonstrated by perimetry.

In 2015, Jabs et al. ${ }^{20}$ defined HIV as a neuroretinal disorder (HIVNRD) in PLHIV, if mean deviations on the 24-2 programme (Humphrey's perimeter) was worse than $-3 \mathrm{~dB}$ and CS was worse than $\log 1.5$. It was found, using this criterion, in a sample with a median CD4 count of 178 cells / $\mathrm{mm}^{3}$ that $16 \%$ were classified with HIVNRD. The median mean deviation for those with HIVNRD was $-4.9 \mathrm{~dB}$. These perimetric changes support the argument that HIV is an immune disorder, resulting in the addition of a specific neuroretinal disorder in the absence of retinal or central nervous system (CNS) infections.

The evidence summarised in Table 2 suggests that perimetric changes in PLHIV without retinopathy occurs at a CD4 count of less than 200 cells $/ \mathrm{mm}^{3}$. This manifests as reduced mean deviations by at least $2 \mathrm{~dB}$ in the central 24 degrees of the visual field, and local field changes manifest by changes occurring in PSD and GHT. This suggests that visual fields are generally depressed as local defects, and in specific areas of vulnerability, as sub-served by the GHT. The magnitude of perimetric changes may appear subtle but over time this may threaten vision loss. Studies of PLHIV with CD4 counts of above 500 are not included in this review.

Contrast sensitivity in people living with HIV: The value of assessing CS over plain visual acuity is that CS measures visual performance at varying contrasts and spatial frequencies while visual acuity is limited to high spatial frequencies (superior vision) at a single contrast level. This therefore makes CS assessment a more sensitive evaluation of vision. Contrast sensitivity in PLHIV has been investigated primarily using two techniques: the Vistech CS Test which was mostly used in the 1990s and, more recently, the PelliRobson Chart.

The Mueller et al. ${ }^{19}$ study, described earlier, recorded a general loss of CS amongst individuals and showed that amongst PLHIV there was a correlation between CS and perimetry and between CS and colour vision.

The 2000s marked the start of the common use of the Pelli-Robson CS Test. Studies ${ }^{13,21}$ on HIV and CS using the Pelli-Robson Chart found a median CS log of 1.65 and concluded that in PLHIV the CS was abnormal for scores of less than $\log 1.5$, benchmarking and providing empirical evidence for CS in PLHIV for subsequent studies. Using this criterion, Shah et al. ${ }^{21}$ found $7 \%$ of PLHIV with abnormal CS and, furthermore, reported that abnormal colour vision and CS can occur independently in these patients. Freeman et al. ${ }^{13}$ found $12 \%$ of PLHIV with CD4 counts of less than 200 cells $/ \mathrm{mm}^{3}$ with abnormal CS in PLHIV. In addition to Shah et al., ${ }^{21}$ the study findings with regards to CS were that there was a correlation between diminishing CS and decreasing levels of CD4 count levels (180 cells $/ \mathrm{mm}^{3}$ ).

In 2015, Jabs et al. ${ }^{20}$ reported findings from a longitudinal study of the ocular complications of AIDS (LSOCA) between 1998 and 2013, and reported on CS abnormalities amongst PLHIV. These researchers asserted that the abnormalities should be defined as HIVNRD when visual field assessments showed mean deviations that were worse than $-3 \mathrm{~dB}$ and CS tests produced scores that were worse than $\log 1.5$. Using these terms of reference, $16 \%$ of the study sample could be classified as exhibiting HIVNRD and, notably, the visual field functionality of these individuals was more reduced. Importantly, the study reported that the risk of HIVNRD doubled when PLHIV had CD4 counts of less than 100 cells $/ \mathrm{mm}^{3}$.

In 2012, Holland et al.22 concluded that abnormal CS was associated with increased mortality and is an independent risk factor for death amongst PLHIV in view of the association with microvascular pathology, similar to diabetes mellitus. Eight percent of the sample was assessed to have abnormal CS, using a median CS of log 1.65.

Table 2 provides quantitative evidence that CS is affected in PLHIV on ART without retinopathy and with CD4 counts of 
less than 200 cells $/ \mathrm{mm}^{3}$, and that this can be evaluated when the Pelli-Robson score is less than $\log 1.5$. There is a dearth of evidence amongst PLHIV with high immunocompetence in an age where ART is lengthening the lifespan of PLHIV, which questions if these observed changes will increase over time.

Colour vision in people living with HIV: The evidence for the prevalence of colour vision loss amongst PLHIV is limited and broad in terms of its findings. This may be owing to the differences in the types of tool used to test colour vision and the varied ranges of CD4 counts and immunocompetence. The tools used include the anomaloscope, which is a colourmatching test that utilises illumination of three spectral sensitivities, viz. red, green and yellow, based on the long-, medium- and short photoreceptor cones in the eye to detect normal matches. The other test is the Farnsworth-Munsell (FM) Test, which is a pigment colour arrangement test that utilises caps that need to be arranged sequentially according to the visible spectrum of light. The studies using the Farnsworth-Munsell 100 Hue (FM100) version of the test analysed the root mean square of the total error score to gauge severity, whereby an increase in this magnitude is regarded as poor colour vision. Both techniques together can assess various colour defects and colour acuity.

Sommerhalder et al., ${ }^{23}$ using the anomaloscope, found no significant colour vision anomalies in PLHIV with no retinitis at a median CD4 count of 70 cells $/ \mathrm{mm}^{3}$. In contrast, Mueller et al., ${ }^{19}$ using the FM100 Test, reported that $29.4 \%$ of the individuals had abnormal colour vision, with the root mean square of the total error score being 8.31 (normal = 5.16) with a median CD4 count of 173 cell $/ \mathrm{mm}^{3}$. However, Shah et al., ${ }^{21}$ in the mid-2000s, using the same test and on a similar sample with a median CD4 count of 330 cell $/ \mathrm{mm}^{3}$, reported that $9.9 \%$ of the sample had abnormal colour vision with the root mean square of the total error score being 10.20. These FM100 Test studies indirectly show that as the CD4 count increases, the prevalence of colour vision loss is reduced but without an axis of defect, therefore suggesting no predilection to a particular cone dysfunction but a general depression in retinal function. ${ }^{24}$

The changes in colour vision suggest the involvement of the photoreceptor, which therefore extends retinal involvement to the outer retina and beyond the RNFL. Kozak et al. ${ }^{24}$ refuted the categorical involvement of the inner retina alone, as shown with RNFL, by stating that photoreceptor dysfunction also implied outer retinal involvement in PLHIV. Table 2 quantitatively allows for the conclusion that colour vision is affected in PLHIV without retinitis, and manifested a higher root mean square of the total error scores of above 8 on the FM100.

In summation, it is clear that among PLHIV on ART without retinitis, the psychophysical visual function is affected but is limited to CD4 counts of between 200 cells $/ \mathrm{mm}^{3}$ and $500 \mathrm{cell} / \mathrm{mm}^{3}$. However, the identified gap in the literature should examine whether or not these changes extend to a higher level of immunocompetence where there is viral suppression and CD4 counts of greater than 500 cells $/ \mathrm{mm}^{3}$. The question still to be answered is whether the maintenance of ART and the elevation of CD4 count actually guard against further deterioration, or if the compounded presence of the virus over time affects the tissues of the eye and threatens vision loss in light of the extended lifespan expectation of PLHIV.

\section{Retinal nerve fibre layer and visual function in people living with HIV}

There is an association between RNFL loss and visual function loss in PLHIV on ART without retinitis. Table 3 shows studies that relate the structural (RNFL) and functional (vision) relationship in PLHIV on ART without retinitis. The glaucoma model aptly shows that in early disease states there are small retinal ganglion cell (RGC) changes and larger RNFL changes corresponding to small changes in the mean deviation on automated perimetry. While at advanced states, changes in retinal ganglion cells showed larger changes in mean deviation and smaller changes in mean deviation for RNFL. This relationship supports the premise that diseases affecting the RNFL directly affect vision. In this case, HIV, albeit a different mechanism, as shown in Tables 1 and 2, demonstrates the structural retinal changes and visual functional changes that occur in PLHIV. The magnitude of these changes may not translate into quality of life changes; however, the studies in Table 3 show concurrent RNFL thinning in the presence of visual loss such as perimetry, CS and colour vision in PLHIV on ART without retinitis.

\section{Retinal nerve fibre layer and perimetry in people living with HIV}

Despite limited studies, the attempt to relate visual field changes with RNFL changes is evident. Studies in glaucoma have shown that there is an association between RNFL, the ganglion cell layer and perimetric changes. Perimetric assessment using either Humphrey's frequency doubling technique (FDT) or short wavelength automated perimetry (SWAP) is influenced by RNFL and changes in ganglion cell thickness.

Arantes et al. ${ }^{25}$ managed to find RNFL thinning nasally, with FDT perimetric loss in PLHIV with CD $4<100$ cells $/ \mathrm{mm}^{3}$. It showed the low CD4 count group to have a reduction in average ppRNFL thickness with the exception of the temporal zone but also showed thinning in the temporal and inferior outer macula zones. All this occurred with concurrent reduced values in the MD and the PSD. It was further shown that the eyes with affected PSD and GHT outside normal limits had a thinner average ppRNFL in PLHIV on ART without retinitis. Arantes et al. ${ }^{26}$ went on in 2012 to specifically show independent thinning in the ppRNFL in the nasal, inferior and temporal zones, respectively. Independent defects in the nasal visual field zones also occurred. An association between the PSD and the average ppRNFL was shown and the strongest correlations occurred between the 
superior ppRNFL and inferior visual field, and the nasal ppRNFL and the temporal visual field in PLHIV on ART without retinitis.

A noteworthy observation is the use of the FDT perimeter in these studies, which has been shown to target ganglion cells of the magnocellular pathway and has the ability to predict field loss before standard automated perimetry. This is significant in light of the fact that PLHIV on ART without retinitis are the focus of this review. Table 3 provides quantitative evidence which concludes that reduced MD, PSD and GHT are all affected concurrently with a thinning of ppRNFL in PLHIV on ART without retinitis at reduced CD4 counts of 100 cell $/ \mathrm{mm}^{3}$.

\section{Retinal nerve fibre layer and contrast sensitivity/visual acuity/colour vision in people living with HIV}

Kalyani et al. ${ }^{12}$ noted temporal ppRNFL loss and reduced CS in PLHIV for more than 180 months, which shows a positive correlation. Pathai et al. ${ }^{27}$ specifically showed an association of lower CS at $\log 1.70$ and a thinner temporal ppRNFL. Most recently, Paul et al. ${ }^{28}$ also found a positive correlation of reduced CS at $\log 1.33$ with the temporal ppRNFL.

Gender bias may not be an influence, as the sample profile used by Kalyani et al. ${ }^{12}$ was predominantly male and that of Pathai et al. ${ }^{27}$ was predominantly female, and both results showed a favourable agreement. Furthermore, the study by Pathai et al. ${ }^{27}$ was geographically located in South Africa, while Paul et al. ${ }^{28}$ studied a sample in India and also found similar results, suggesting that race and geography may not be an influencer. Table 3 provides evidence and allows for the conclusion: that the temporal ppRNFL is associated with reduced CS in PLHIV on ART without retinitis. However, the agreement of the magnitudes of the reduced CS and the temporal ppRNFL still requires more investigation.

The review for the assessment of visual acuity and the RNFL in PLHIV on ART without retinitis is limited to Barteselli et al..$^{29}$ who showed for patients with CD4 counts of less than 100 cells $/ \mathrm{mm}^{3}$ a loss of inferior-temporal ppRNFL, for varying contrasts and illumination. The study suggests that the HIV status independently predicts visual performance under varying contrasts at $100 \%, 64 \%$ and $43 \%$. Accordingly, visual acuity using the Early Treatment Diabetic Retinopathy Study (ETDRS) charts (logMAR acuity) at varying contrasts behaves in a similar way to CS, but both showed temporal RNFL associations in PLHIV on ART without retinitis.

The review for the assessment of colour vision and the ppRNFL in PLHIV on ART without retinitis is limited to Kalyani et al. ${ }^{12}$ This study showed an inverse correlation of the temporal ppRNFL with the colour confusion index (CCI) for the Lanthony D15. Higher CCI indices suggest poorer colour acuity, which occurred with the thinning of the temporal RNFL in PLHIV on ART without retinitis. It is important to remember that colour vision involvement extends beyond the RNFL because the photoreceptors lie in the outer retina.
Table 3 provides the evidence for HIV causing ppRNFL loss at low CD4 counts, and that the visual functions at risk are CS, colour vision and visual fields concurrently. Most specifically, similar correlations for CS, visual acuity and colour vision changes with the temporal ppRNFL were established. However, the majority of the studies limit interest to CD4 $<200$ cells $/ \mathrm{mm}^{3}$ and to an area of visual function that is sub-clinical. More research is required in PLHIV with elevated CD4 counts regarding visual function and the manner in which this has an impact on daily functioning in light of PLHIV living longer. Consequently, this threat of vision loss needs addressing.

\section{Neuro-ophthalmological associations in HIV in an era of antiretroviral therapy}

Table 4 groups studies that show the neuro-ophthalmological relationship between the retina and the brain in HIV in the era of ART. Although some are not statistically significant observations, there are patterns of relationships. They may not be clinically significant but do show changes in the absence of prospective studies.

The ophthalmic arm of the AGEhIV (neuroretinal changes in HIV-positive adults) has most recently reported that adults with prolonged and suppressed viremia whilst on ART do not appear to be at risk of developing HIV neuroretinal disorders and suggested that there is an undisrupted physiological retina-brain correlation. However, the study could not conclude that the retinal OCT may be a useful marker to specifically indicate HIV-related neuroretinal degeneration.

Demirkaya et al. ${ }^{30}$ relate the retina to grey and white matter changes in HIV in the era of ART. Total grey matter volume was positively correlated to foveal GCL, pericentral total retinal thickness, RNFL and GCL, peripheral total retinal thickness, RNFL and inner plexiform layer (IPL) thickness. Cortical white matter was positively correlated to foveal and pericentral GCL, and peripheral RNFL thickness. Further to this, on magnetic resonance diffusion imaging they showed significant positive correlations between pericentral retinal thickness - in particular of the inner layers - and fractional anisotropy (FA), and negative associations between the same layers and mean diffusivity (MD). All these were observed in virally suppressed PLHIV on ART and are the findings of the AGEhIV study which demonstrates that the retina and brain are disrupted in the age of ART.

However, the AGEhIV study assessed virally suppressed men older than 45 years and concluded that retinal thickness and cerebral parameters were similar to those of normal people. Haddow et al. ${ }^{31}$ also attempted to biomark retinal vessels for cerebrovascular ageing in PLHIV; however, no differences in retinal vascular indices in HIV-positive and HIV-negative men over 50 years were noted.

Blokhuis et al..$^{32}$ studied children with HIV on ART who were virally suppressed and found that reduced neuroretinal 
thickness was associated with microstructural white matter injury. Thereafter, Blokhuis et al., ${ }^{33}$ in a sample, inversely correlated blood plasma levels of IL-6, MCP-1 and sICAM1 with foveal GCL thickness. Neuronal biomarkers such as cerebrospinal fluid (CSF) tTau levels inversely correlated with pericentral total retinal thickness and ONL/IS in both the foveal and pericentral regions. The relevance of these highlights biomarkers associated with inflammatory processes and neurodegenerative disease, and may help to explain observations in virally suppressed PLHIV. All these are the findings of the study by NOVICE (neuroretinal changes in HIV-positive children) and also demonstrates neuro-ophthalmological compromise in the present age of ART.

The NOVICE study looked at HIV-positive children between 8 and 18 years of age and reported that a decrease in foveal thickness was associated with a higher peak viral load. Further to this, it found that retinal thickness was associated with microstructural white matter injury in HIV infection in children. However, no significant changes occurred in colour vision, central visual field and CS function, but a thinner total foveal thickness occurred, caused by a thinning of the outer nuclear layer and inner segment. They attributed this to perinatal infection disturbing foveal maturation which did not disturb visual function. Furthermore, retinal thickness was associated with microstructural white matter injury in HIV-positive children by showing lower FA, higher MD and higher radial diffusion on diffusion-weighted imaging. However, work by Crowell et al. ${ }^{34}$ did establish that virologic suppression during infancy or early childhood was associated with improved neurocognitive outcomes in school-aged children.

Much earlier, in 2012, Jesus-Acosta et al..$^{35}$ associated the RNFL to brain dysfunction and neurodegeneration in HIV (HAND) and highlighted the OCT as a tool that could represent a biomarker for HIV-associated neurocognitive disorder. Together with the AGEhIV and NOVICE studies, these make a case that in the era of ART there still remains a threat from the virus or, more controversially, the treatment, as PLHIV live longer.

The discrepancy in the NOVICE and AGEhIV findings was attributed to the continuous development of children, and that unsuppressed HIV in their early years may affect maturation. The findings of these studies may not be clinically relevant; however, the trace of the virus from the retina to the brain did show changes. As a result of the extended lifespans of PLHIV on ART, if followed prospectively, these findings may become clinically relevant. This may be the beginning of the recognition of the threat to vision and the brain in virally suppressed PLHIV.

\section{Antiretroviral therapy and the retina}

Evidence that ART is associated with retinal toxicity exists. Case reports identify ritonavir as a cause of retinal toxicity as a bull's eye maculopathy and, in some cases, this mimics retinitis pigmentosa. ${ }^{36,37}$ Faure et al. ${ }^{37}$ found that the toxicity continues despite cessation of ritonavir. Bull's eye retinopathy has also been identified in PLHIV with elevated CD4 counts and low viral loads using efavirenz, lamivudine and zidovudine. ${ }^{38}$ The systemic effects of ART that may lead to retinal changes may be a reality as a consequence of atherosclerotic vascular changes related to ART that elevate lipid levels and in some cases cause the occlusion of the central retinal artery. ${ }^{39}$ Antiretroviral therapy has been found to also cause metabolic toxicity to beta cell function, affecting insulin function, and glucose levels, ${ }^{40}$ which may then affect the vascular integrity of the retina. The vascular impact of ART on the retina should be factored into studies going forward when studying PLHIV on ART who are virally suppressed, especially in light of the extended lifespan these persons are now being afforded.

\section{Conclusion}

The decrease in visual function and ppRNFL loss, in PLHIV on ART without retinitis who were flagged in this review, allow us to recognise the threat to vision loss. The affected visual functions include perimetry, CS, colour vision and visual electrophysiology in PLHIV on ART without retinitis. The structural indicator that is affected is the mean or overall ppRNFL thickness, with the superior and inferior ppRNFL zones both showing thinning. Over time, this can be translated to suggest atrophy that may affect function. These changes may be attributed to the disruption of microvascular circulation, cytokine release syndrome (apoptosis) and accelerated ageing. The RNFL involvement is part of a wider neuroretinal disorder that involves axonal loss. Studies on neuro-ophthalmological changes in an era of ART did find statistically significant changes in retinal layers and cerebral volume, which did not manifest clinically but established neuro-ophthalmic disruption anatomically. Further to this, one cannot discount the extenuating circumstance of the role of ART itself on the retina, which may also be a contributing factor that must be accounted for in future studies. All these changes flagged in the literature should be recognised as a threat to vision and the brain when factoring in the expected longer lives that PLHIV are afforded with ART.

\section{Acknowledgements}

The authors would like to acknowledge the writing mentoring workshop of the African Journal of AIDS Research (AJAR) and the facilitation in mentoring the corresponding author with the article construction.

\section{Competing interests}

The authors declare that they have no competing interests.

\section{Authors' contributions}

A.J.M. is the lead investigator. A.A.M. and R.L.B. are the supervisors of the project. All authors read and approved the final manuscript. 


\section{Funding information}

Funding was received from the University of KwaZuluNatal's Developing Research Innovation, Localisation and Leadership in South Africa (DRILL) programme. This programme is funded by the Fogarty International Center of the National Institutes of Health under Award Number D43TW010131.

\section{Data availability statement}

Data sharing is not applicable to this article.

\section{Disclaimer}

The content is solely the responsibility of the authors and does not necessarily represent the official views of the National Institutes of Health.

\section{References}

1. Avert. HIV and AIDS in South Africa [homepage on the Internet]. [cited 2019 Nov 06] Available from: https://www.avert.org/professionals/hiv-around-world/subsaharan-africa/south-africa

2. Plummer DJ, Bartsch D-UG, Azen SP, Max S, Sadun AA, Freeman WR. Retinal nerve fiber layer evaluation in human immunodeficiency virus - positive patients. Am J Ophthalmol. 2001;131(2):216-222. https://doi.org/10.1016/S0002-9394(00) 00787-X

3. Kozak I, Bartsch D-U, Cheng L, Kosobucki BR, Freeman WR. Objective analysis of retinal damage in HIV-positive patients in the HAART era using OCT. Am Ophthalmol. 2005;139(2):295-301. https://doi.org/10.1016/j.ajo.2004.09.039

4. Kozak I, Bartsch D-UG, Cheng L, McCutchan A, Weinreb RN, Freeman WR. Scanning laser polarimetry demonstration of retinal nerve fiber layer damage in human immunodeficiency virus - positive patients without infectious retinitis. Retina. 2007;27(9):1267-1273. https://doi.org/10.1097/IAE.0b013e31806463fb

5. Besada E, Shechtman D, Black G, Hardigan PC. Laser scanning confocal ophthalmoscopy and polarimetry of human immunodeficiency virus patients without retinopathy, under antiretroviral therapy. Optom Vis Sci. 2007; 84(3):189-196. https://doi.org/10.1097/OPX.0b013e31803399f3

6. Arcinue CA, Bartsch D-U, El-Emam SY, et al. Retinal thickening and photorecepto loss in HIV eyes without retinitis. PLoS ONE. 2015;10(8):e0132996. https://doi. org/10.1371/journal.pone.0132996

7. Tenhula WN, Xu S, Madigan MC, Heller K, Freeman WR, Sadun AA. Morphometric comparisons of optic nerve axon loss in acquired immunodeficiency syndrome. Am J Ophthalmol. 1992;113(1):14-20. https://doi.org/10.1016/S0002-9394(14) 75746-0

8. Deeks SG. Immune dysfunction, inflammation, and accelerated aging in patients on antiretroviral therapy. Top HIV Med. 2009;17(4):118-123.

9. Capeau J. Premature aging and premature age-related comorbidities in HIVinfected patients: Facts and hypotheses. Clin Infect Dis. 2011;53(11):1127-1129. https://doi.org/10.1093/cid/cir628

10. Demirkaya N, Wit F, Schlingemann R, Verbraak F. Neuroretinal degeneration in HIV patients without opportunistic ocular infections in the CART era. AIDS Patient Care STDS. 2015;29(10):519-532. https://doi.org/10.1089/apc.2015.0091

11. Pelegrin Colas L, Bitrian E, Casaroli-Marano R, et al. Changes of retinal nerve fiber layer (RNFL) thickness in patients with human immunodeficiency virus (HIV) treated with HAART: An OCT study. Invest. Ophthalmol. Vis. Sci. 2007;48(13):342. http://iovs.arvojournals.org/article. aspx?articleid=2382546.

12. Kalyani PS, Holland GN, Fawzi AA, Arantes TEF, Yu F, Sadun AA. Association between retinal nerve fiber layer thickness and abnormalities of vision in people with human immunodeficiency virus infection. Am J Ophthalmol. 2012;153(4):734-742, 742.e1. https://doi.org/10.1016/j.ajo.2011.09.019

13. Freeman WR, Van Natta ML, Jabs D, et al. Vision function in HIV-infected individuals without retinitis: Report of the studies of ocular complications of AIDS Research Group. Am J Ophthalmol. 2008;145(3):453-462. https://doi.org/10.1016/j.ajo.2007. 10.013

14. Szanyi J, Kremlacek J, Kubova Z, et al. Pattern- and motion-related visual evoked potentials in HIV-infected adults. Doc Ophthalmol. 2017;134(1):45-55. https:// doi.org/10.1007/s10633-016-9570-x

15. Falkenstein I, Kozak I, Kayikcioglu O, et al. Assessment of retinal function in patients with HIV without infectious retinitis by multifocal electroretinogram and automated perimetry. Retina. 2006;26(8):928-934. https://doi.org/10.1097/01. iae.0000250009.60908.35

16. Iragui VJ, Kalmijn J, Plummer DJ, Sample PA, Trick GL, Freeman WR. Pattern electroretinograms and visual evoked potentials in HIV infection: Evidence of asymptomatic retinal and postretinal impairment in the absence of infectious retinopathy. Neurology. 1996;47(6):1452-1456. https://doi.org/10.1212/WNL. 47.6.1452
17. Falkenstein IA, Bartsch D-U, Azen SP, Dustin L, Sadun AA, Freeman WR. Multifocal electroretinography in HIV-positive patients without infectious retinitis. Am J Ophthalmol. 2008;146(4):579-588. https://doi.org/10.1016/j. ajo.2007.12.021

18. Goldbaum MH, Falkenstein I, Kozak I, et al. Analysis with support vector machine shows HIV-positive subjects without infectious retinitis have mfERG deficiencies compared to normal eyes. Trans Am Ophthalmol Soc. 2008;106 (106):196-205.

19. Mueller AJ, Plummer DJ, Dua R, et al. Analysis of visual dysfunctions in HIVpositive patients without retinitis. Am J Ophthalmol. 1997:124(2):158-167. positive patients without retinitis. Am Joi.org/10.1016/S0002-9394(14)70780-9

20. Jabs DA, Drye L, Van Natta MHS, Thorne JE, Holland GN. Incidence and long-term outcomes of the human immunodeficiency virus neuroretinal disorder in patients with AIDS. Ophthalmology. 2015;122(4):760-768. https://doi.org/10.1016/j. with AlDS. Ophtha.2014.11.009

21. Shah KH, Holland GN, Yu F, Van Natta M, Nusinowitz S. Contrast sensitivity and color vision in HIV-infected individuals without infectious retinopathy. Am Ophthalmol. 2006;142(2):284-292. https://doi.org/10.1016/j.ajo.2006.03.046

22. Holland GN, Kappel PJ, Van Natta ML, et al. Association between abnormal contrast sensitivity and mortality among people with acquired immunodeficiency syndrome. Am J Ophthalmol. 2010;149(5):807-816. https://doi.org/10.1016/j. ajo.2009.12.019

23. Sommerhalder J, Baglivo E, Barbey C, Hirschel B, Roth A, Pelizzone M. Colour vision in AIDS patients without HIV retinopathy. Vision Res. 1998;38(21):3441-3446. https://doi.org/10.1016/S0042-6989(98)00011-X

24. Kozak I, Sasik R, Freeman WR, et al. A degenerative retinal process in HIVassociated non-infectious retinopathy. PLoS ONE. 2013;8(9):e74712. https://doi. org/10.1371/journal.pone.0074712

25. Arantes TEF, Garcia CR, Mello PA de A, Muccioli C. Structural and functional assessment in HIV-infected patients using optical coherence tomography and frequency-doubling technology perimetry. Am J Ophthalmol. 2010;149(4): 571-576. https://doi.org/10.1016/j.ajo.2009.11.026

26. Arantes TE, Garcia CR, Tavares IM, Mello PA, Muccioli C. Relationship between retinal nerve fiber layer and visual field function in human immunodeficiency virus-infected patients without retinitis. Retina. 2012;32(1):152-159. https://doi. org/10.1097/IAE.0b013e31821502e1

27. Pathai S, Lawn SD, Weiss HA, Cook C, Bekker L-G, Gilbert CE. Retinal nerve fibre layer thickness and contrast sensitivity in HIV-infected individuals in South Africa: A case-control study. PLoS ONE. 2013;8(9):e73694. https://doi.org/10.1371/ journal.pone.0073694

28. Paul R, Ghosh AK, Nag A, Biswas S, Naiya B, Mondal J. Study of retinal nerve fibre layer thickness and visual contrast sensitivity in HIV positive individuals. J Clin Diagnostic Res. 2017;11(6):OC01-OC04. https://doi. org/10.7860/JCDR/2017/24751.9956

29. Barteselli G, Chhablani J, Gomez ML, et al. Visual function assessment in simulated real-life situations in HIV-infected subjects. PLoS ONE. 2014;9(5):e97023. https:// doi.org/10.1371/journal.pone.0097023

30. Demirkaya N. In search of retinal biomarkers for HIV related neurodegeneration The AGEhIV and NOVICE studies. Holland: University of Amsterdam; 2018.

31. Haddow L, Laverick R, Leung I, et al. Measurement of retinal vessels as a biomarker of cerebrovascular aging in older HIV-positive men compared with controls. Acquir Immune Defic Syndr. 2018;77(2):199-205. https://doi.org/10.1097/qai. 0000000000001570

32. Blokhuis C, Demirkaya N, Cohen S, et al. The eye as a window to the brain Neuroretinal thickness is associated with microstructural white matter injury in HIV-infected children. Investig Opthalmology Vis Sci. 2016;57(8):3864-3871. https://doi.org/10.1167/iovs.16-19716

33. Blokhuis C, Doeleman S, Cohen S, et al. Inflammatory and neuronal biomarkers associated with retinal thinning in pediatric HIV. Investig Opthalmology Vis Sci. 2017;58(13):5985-5992. https://doi.org/10.1167/iovs.17-22252

34. Crowell C, Huo Y, Tassiopoulos K, et al. Early viral suppression improves neurocognitive outcomes in HIV-infected children. AIDS. 2015;29(3):295-304 https://doi.org/10.1097/QAD.0000000000000528

35. De Jesus-Acosta C, Rosado N, Hechavarria $R$, et al. Thinning of retinal nerve fiber layer is associated with neurocognitive impairment in HIV infected women. Neurology. 2012;78:P01.263-P01.263. https://doi.org/10.1212/WNL.78.1_ MeetingAbstracts.P01.263

36. Papavasileiou E, Younis S, Zygoura V, Quijano C, Jackson TL. Ritonavir-associated toxicity mimicking retinitis pigmentosa in an HIV-infected patient on highly active antiretroviral therapy. Retin Cases Brief Rep. 2017;11(4):306-309. https://doi. org/10.1097//CB.0000000000000350

37. Faure $C$, Paques $M$, Audo I. Electrophysiological features and multimodal imaging in ritonavir-related maculopathy. Doc Ophthalmol. 2017;135(3):241-248. https:// doi.org/10.1007/s10633-017-9612-z

38. Martin-Odoom A, Bonney EY, Opoku DK. Ocular complications in HIV positive patients on antiretroviral therapy in Ghana. BMC Ophthalmol. 2016;16:134. https://doi.org/10.1186/s12886-016-0310-5

39. Lenci LT, Chin EK, Almeida DRP. Central retinal artery occlusion in a young HIVinfected patient on highly active antiretroviral therapy. Retin Cases Brief Rep. 2017;11(2):160-162. https://doi.org/10.1097/ICB.0000000000000318

40. Sims EK, Park G, Mather KJ, Mirmira RG, Liu Z, Gupta SK. Immune reconstitution in ART treated, but not untreated HIV infection, is associated with abnormal beta cell function. PLoS ONE. 2018;13(5):e0197080. https://doi.org/10.1371/journal. pone.0197080 